\title{
Neurociencia y TEL: el desconocimiento docente.
}

(Neuroscience and SLD: teacher ignorance.)

\author{
María Moreno Maestre \\ Universidad de Jaén (España) \\ mariam9811@gmail.com
}

Fecha recepción: 12-04-2021

Páginas 101-109

Fecha aceptación: 25-05-2021

\section{Resumen.}

El presente trabajo se lleva a cabo con el fin de revisar y analizar los resultados de estudios publicados sobre neurociencia y Trastornos Específicos del Lenguaje y/o disfasia. Se plantea la neurociencia como una herramienta eficaz para la detección e intervención de estas patologías, con el fin de conseguir una respuesta educativa integradora e inclusiva. Tras la investigación de dichos artículos, se ha comprobado que, los docentes no poseen conocimientos acerca de la neurociencia implementada en la educación, como estrategia eficaz para saber cómo funciona el cerebro del alumnado, y, por ende, cómo aprende. Además, se ha verificado que, no conocen los aspectos que pueden influenciar en posibles casos TEL o disfásicos, ni cómo llevar a cabo una intervención educativa. Por ello, surge la necesidad de ahondar en el tema para la detección temprana de los mismos.

Palabras clave: neurociencia; Trastorno Específico del Lenguaje; disfasia; lenguaje; docentes; inclusión

\begin{abstract}
.
The present work is carried out in order to review and analyze the results of studies published on neuroscience and Specific Disorders of Language and/or dysfasia. Neuroscience is proposed as an effective tool for the detection and intervention of these pathologies, in order to achieve an inclusive and inclusive educational response. After researching these articles, it has been found that teachers do not have knowledge about the neuroscience implemented in education, as an effective strategy to know how the brain of students works, and, therefore, how they learn. In addition, it has been verified that they do not know the aspects that can influence in possible cases TEL or dysphasic, or how to carry out an educational intervention. Therefore, the need arises to delve into the topic for the early detection of them.
\end{abstract}

Keywords: neuroscience; Language Specific Disorder; dysphasia; language; teachers; inclusion 


\section{1.-Introducción.}

La neurociencia se define como: "Ámbito de conocimiento científico que estudia los distintos aspectos del sistema nervioso de modo unificado: el desarrollo, la estructura 0 el funcionamiento. Su objetivo es explicar los diferentes componentes del sistema nervioso" (Portellano, 2018, p. 14). Esta disciplina ha evolucionado y cogido impulso en los últimos años.

Este término ha supuesto una gran revolución en el ámbito educativo. Sin embargo, como alegan Román y Poenitz (2018), se hace necesario cuestionarse en qué medida influye en las praxis eficientes e interdisciplinares a la propia naturaleza de la formación. Actualmente, la neurociencia está ganando terreno en las aulas, por ello, que surge el término enlace entre ambos conceptos como una propuesta para los docentes y expertos en el área de la enseñanza: "neuroeducación". Portellano (2018), la describe de la siguiente manera: "es una disciplina que integra los principios de educación y neurociencia, aquella que se basa en los componentes o funcionamiento del SNC para comprender la conducta humana en el proceso de enseñanza" (p. 9).

A día de hoy, existe poca fundamentación teórica relacionada con la disciplina mencionada, lo que repercute en el saber de los docentes sobre la misma. Como cita Morán (2020): "La neuroeducación se pretende introducir en las aulas de las escuelas, para potencializar las prácticas pedagógicas, proporcionando espacios dinámicos y diversos a los alumnos para construir su aprendizaje" (p. 45). Esta labor no solo delega en estos maestros y maestras, sino, también, de todas las familias del alumnado. En definitiva, se plantea como una nueva forma de mejorar la educación.

Ahondando más en el tema, encontramos el término de neurodidáctica. Según Morán (2020), se define como: "Campo de la pedagogía basada en la neurociencia que tiene el objetivo de buscar una nueva dirección para el diseño de estrategias didácticas más eficientes, las cuales promuevan un mayor aprendizaje conforme al desarrollo cerebral" (p. 1). De esta manera, se construyen aprendizajes conforme al desarrollo evolutivo del cerebro del alumnado.

Como ya hemos citado anteriormente, la neurociencia es una disciplina que está en auge desde no hace mucho tiempo. Es por ello que, siguen surgiendo ideas y creencias sobre la veracidad de la misma, apareciendo, así, los neuromitos.

La neurociencia integra una serie de principios en el ámbito educativo, defendiendo que, existen varios factores que hacen que el cerebro se pueda moldear, como pueden ser: el entorno, la genética y la experiencia. (Román y Poenitz, 2018). Por otro lado, llevar a cabo esta disciplina al aula, también supone ciertos riesgos: el paradigma, los propios docentes, los programas de formación o la política. Así los prescriben los autores citados anteriormente. 
Este término "Trastorno Específico del Lenguaje" ha sufrido modificaciones tanto en su nomenclatura, como en el saber de sus causas. Salvatierra y Zambrano (2021), lo definen como una patología propia del desarrollo del habla y del lenguaje, que afecta a la destreza oral.

Esta patología ha sido diferenciada en dos subtipos: TEL expresivo y TEL mixto. El primero, según la Armada de Chile (2019), solo afecta a la parte expresiva del lenguaje, mientras que, el segundo, afecta tanto a nivel expresivo, como a nivel comprensivo.

Por otro lado, existen diferentes categorías que dividen este tipo de trastorno. Centrándonos en la que nos compete y la que va a ser descrita en el presente trabajo, la disfasia se encuentra en el tipo Trastorno del Lenguaje, pudiendo mencionar a la misma vez, las diferentes clasificaciones que existen: alteración del habla, distorsión de los órganos fonoarticulatorios y patologías psicolingüísticas. (Bolte y Rojas, 2015; Artigas, Rigau, y García, 2008;).

Bonilla y Reyes (2017) definen el término disfasia así:

"es un trastorno que es generado por un supuesto daño en el cerebro y, que, produce dificultades en el lenguaje. Por eso, también, se conoce a este estado como trastorno específico del desarrollo del lenguaje. [...] La disfasia es una irregularidad que se detecta en el lenguaje, no puede atribuirse a una discapacidad intelectual, problemas en los sentidos, traumas psicológicos o lesiones cerebrales que resultan evidente". (p. 1).

El concepto de disfasia también ha tenido cambios en su terminología. Anteriormente, se conocía este trastorno como "afasia", señalando la ausencia del lenguaje, mientras que, la disfasia, hace referencia a un trastorno en mayor o menor medida de esta capacidad. (Bonilla y Reyes, 2017, p. 3).

Respecto a las causas o factores que pueden determinar estos trastornos, Auza y Peñaloza (2019) los resumen en tres: el sexo, quedando evidente que el masculino predomina ante la aparición de posibles trastornos de este tipo; la relación entre el trascurso de asistencia 6 escolar de la progenitora y el rendimiento lingüístico del hijo/a en sus primeros años de vida; y, el tiempo que el niño o la niña ha asistido al centro escolar, en este caso, a la etapa de Educación Infantil.

Es cierto que, la detección de estos trastornos no resulta sencilla, pues, suele pasar inadvertida por sus leves manifestaciones a simple vista, y, puede confundirse con un mero retraso del lenguaje propio de la edad. Entre algunos de los síntomas que podemos encontrar, el Instituto Nacional de la Salud (NIH, 2019), cita algunos de ellos: problemas al hablar, inconvenientes a la hora de llevar a cabo una conversación o seguir instrucciones, y redundancia en repetir errores gramaticales, entre otras. De cualquier manera, se hace necesaria la detección e intervención precoz en todos los casos, para de esta manera, evitar que las consecuencias se 
agraven y puedan desencadenar en otros problemas que, anteriormente, no se encontraban.

En última instancia, se pretende conocer algunas estrategias o herramientas para efectuar una intervención eficaz e inclusiva con este tipo de alumnado bajo el prisma de la neurociencia. La Secretaria de Educación Pública de México (2018) recomienda el uso de las Tecnologías de la Información y la Comunicación, más conocidas como TIC. Además de, crear y llevar a cabo situaciones de colaboración con sus iguales, para de esta manera, promover relaciones sociales, y, por último, fomentar el uso de materiales sensoriales que ayuden a involucrar al alumno/a en su desarrollo del aprendizaje.

De aquí, surge la necesidad de revisar este tema, a la vez de explorar, analizar y combinar los resultados de estudios publicados sobre los beneficios que ofrece la neurociencia en alumnos y alumnas que sufren Trastorno Específico del Lenguaje y disfasia.

\section{2.-Neurociencia.}

\section{1.-Concepto.}

El término "neurociencia" se establece como:

"el estudio científico del sistema nervioso (principalmente, el cerebro) y sus funciones. Estudia las complejas funciones de aproximadamente 86 mil millones de neuronas 0 células nerviosas que tenemos. De las interacciones químicas y eléctricas de estas células, las sinapsis, se derivan todas las funciones que nos hacen humanos". (Campos, 2010, p. 48).

\section{2.-Principios.}

En cuanto a los principios de esta disciplina, Andrey (2016) cita los siguientes:

1. La estructura física del cerebro cambia gracias al aprendizaje.

2. Dichos cambios estructurales transforman la organización funcional del cerebro. $E$ aprendizaje organiza y reorganiza el cerebro.

3. Distintas partes del cerebro pueden estar preparadas para aprender en distintos momentos.

4. El cerebro es un órgano activo que se moldea, mayoritariamente, por la experiencia.

En palabras de Andrey (2016), los hallazgos de la Neurociencia nos ayudan a comprender mejor los procesos de enseñanza y aprendizaje de nuestros alumnos y alumnas, y, en consecuencia, a enseñarles de manera más apropiada, efectiva y agradable. Por ello, es de vital importancia que, se siga investigando sobre la misma para la mejora de la práctica docente, pues, la neurociencia, es una disciplina que ha sido considerada desde años remotos bajo el prisma de la biología, pero, lo cierto es 
que, hasta no hace muchos años no se había abordado desde el ámbito de la educación.

\section{3.-TEL.}

\section{1.-Definición.}

Según diversos autores, es una afección que se da en párvulos en paréntesis de la evolución habitual de las habilidades del lenguaje (Bolte y Rojas, 2015), en el ámbito académico es estimada como una dificultad del aprendizaje con alta posibilidad genética (Uzcátegui et al., 2007) con mayor potencial en poblaciones reducidas que se encuentran aisladas, (Villanueva et al., 2008) detectada, asiduamente, en las clínicas pediátricas (Barragán y Lozano, 2011) y en sospecha en los centros docentes.

"Desde un enfoque médico se lo considera una patología en el desarrollo del habla y del lenguaje, desde el punto de vista educativo es considerado una dificultad en el aprendizaje que afecta la destreza oral o del habla" (Salvatierra y Zambrano, 2021, p. 3).

\section{2.-Tipos.}

En cuanto a su tipologia, la Armada de Chile (2019), los clasifica en dos: TEL expresivo y TEL mixto. Según el autor citado, el TEL expresivo, solo afecta a la parte expresiva del lenguaje, presentado así, una buena comprensión lectora (Armada de Chile, 2019). Mientras que, el trastorno específico del lenguaje mixto se ve alterado tanto a nivel expresivo, como a nivel comprensivo.

\section{3.-Clasificación.}

Según Bolte y Rojas (2015), la clasificación de los Trastornos Específicos del Lenguaje parte de la siguiente manera:

Figura 1

La clasificación del TEL según su origen

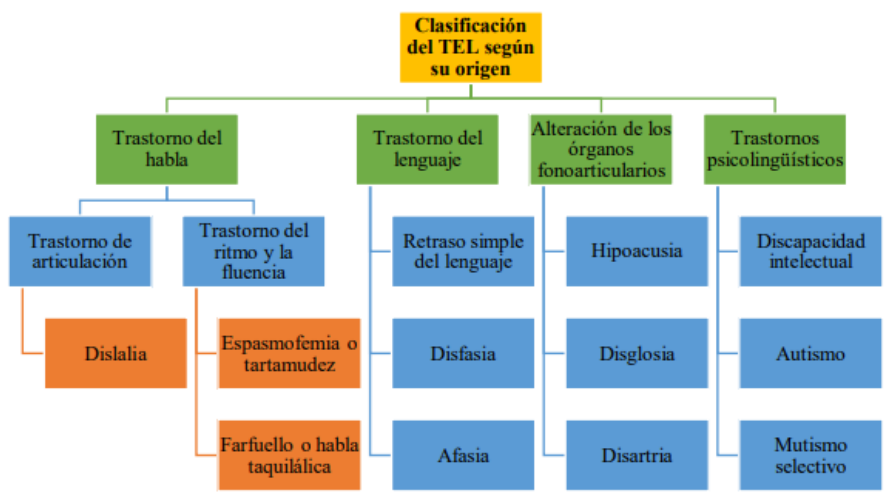


Nota: Adaptado de Clasificación del TEL según su origen, de Bolte y Rojas, 2015; Artigas, Rigau, y García, 2008.

En la anterior figura encontramos varios orígenes sobre el Trastorno Específico del Lenguaje, en este caso, se atención se centrará en los descendientes del lenguaje.

Según Bonilla y Reyes (2017): "el concepto "Disfasia" comenzó a reemplazar al de "Afasia". De acuerdo a la etimología de las palabras, la afasia indicaría la ausencia de lenguaje y la disfasia el trastorno más o menos grave de esta capacidad" (p. 3).

\section{4.-Causas.}

Por último, la casuística de estos transtornos, no se encuentra bajo uma base que sustente cuales son sus verdadeiras causas.

A pesar de ello, a través de distintas investigaciones se ha constatado una mayor vulnerabilidad de presentar el TEL/TDL cuando los sujetos son hombres (Berglund et al., 2005; Choudhury y Benasich, 2003), tienen familiares con algún trastorno de lenguaje. Por otro lado, según Peñaloza (2018), afirma que, una reciente estudio comprobó que, la corta edad de los progenitores, el bajo nivel educativo familia, y la inasistencia a preescolar eran algunos factores asociados a que un niño 0 niña presentara mayor riesgo de poseer esta patología.

\section{4.-Práctica docente.}

Como se ha citado anteriormente, los docentes no tienen conocimientos acerca de estos tipos de transtornos, y, por ende, desconocen cómo llevarlo a la práctica em el aula.

A través de uma encuesta realizada por Bonilla y Reyes (2017) los maestros manifestaron sus conocimientos sobre estas patologías em los siguientes gráficos:

\section{Gráfica 1}

Conocimiento de los docentes acerca de TEL 


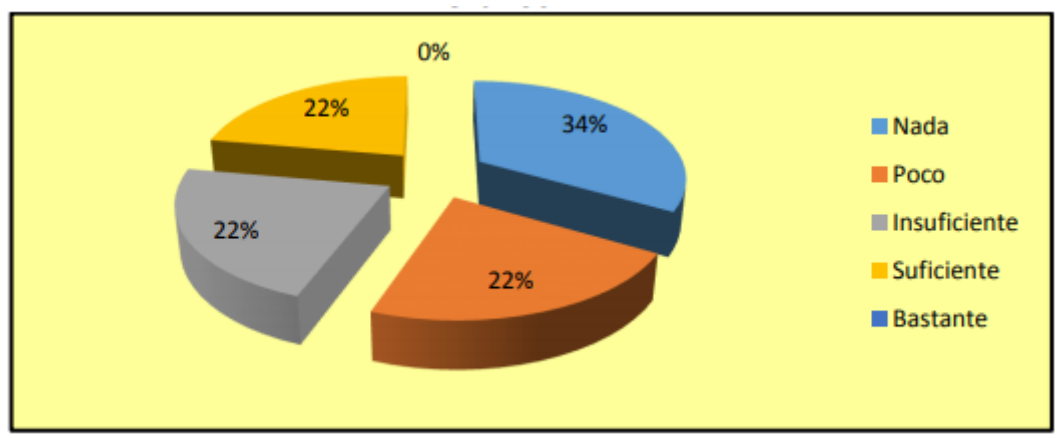

Fuente: Institución Educativa Fiscal "República de Bolivia" Elaborado por: Jennifer Bonilla y Catherine Reyes

Se puede apreciar que, los docentes no están capacitados, por ende, no poseen conocimientos sobre cómo ayudar en la problemática existente que se desarrolla en la institución y los niños y niñas con estas dificultades para expresarse tienen mayor riesgo de desarrollar de forma anómala sus relaciones con los demás.

Así pues, los maestros y maestras consideran que, es necesario llevar a cabo metodologías para el desarrollo expresivo del lenguaje.

\section{Gráfico 2}

Necesidad de implantar metodologias sobre TEL

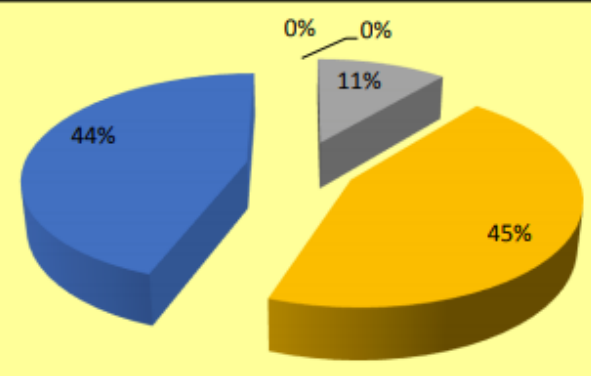

- Innecesario

- Poco necesario

Indiferente

Necesario

Muy necesario

Fuente: Institución Educativa Fiscal "República de Bolivia"

Elaborado por: Jennifer Bonilla y Catherine Reyes

\section{5.-Conclusión.}


Tras el análisis de los diferentes estudios, se puede concluir que, existe gran magnitud de literatura científica que pone de manifiesto resultados favorables sobre la neurociencia implantada en la educación.

Por otro lado, se puede deducir, también, que los docentes no poseen conocimientos acerca de esta especialidad, y, que, por tanto, su aplicación se hace costosa.

Con respecto a los Trastornos Específicos del Lenguaje, se han razonado los factores que pueden incidir en su aparición: el sexo masculino, la escolarización de la madre y el tiempo proporcional del sujeto en el centro escolar. También, el ambiente influye en el agravamiento del mismo, si nos situamos ante un entorno rico en estímulos, el cuadro de diagnóstico será menos agresivo. Además, la colaboración de las familias debe ser primordial como principal fuente de información.

En cuanto a la disfasia, podemos decir que se encuentra bajo el mismo marco que los TEL. Se razona que, una detección precoz y una intervención temprana, pueden ayudar a que este tipo de trastornos no se vea agraviado con el tiempo. Y, que, por tanto, no se asocien otros problemas que no se presentaban anteriormente, que influyan tanto a nivel académico, como a nivel personal.

Finalmente, afirmamos que, tanto los TEL, como la disfasia, si se encuentran bajo una metodología apropiada y acorde a las necesidades de estos alumnos y alumnas, pueden ir desapareciendo poco a poco con el tiempo, por ello, la propuesta de llevar a cabo la neurociencia en el aula como herramienta, sobre todo, para este tipo de situaciones.

\section{6.-Referencias.}

Andrey, M. (2016). Neurociencias y educación. Filial, Lima: Universidad San Pedro.

Artigas, J., Rigau, E., y García-Nonell, K. (2008). Trastornos del lenguaje. AEP: Protocolos de actualización, 24, 178-84.

Auza, A., y Peñaloza Castillo, C. (2019). Factores individuales y familiares en el Trastorno del Desarrollo del Lenguaje (TDL). Iztapalapa. Revista de ciencias sociales y humanidades, 40(86), 41-66. https://doi.org/10.28928/ri/862019/atc2/auzaa/penalozacastilloc

Barragán, P. E., y Lozano, S. S. (2011). Identificación temprana de trastornos del lenguaje. Revista Médica Clínica Las Condes, 22(2), 227-232. https://doi.org/10.1016/S0716-8640(11)70417-5

Berglund, E. V. A., Eriksson, M., y Westerlund, M. (2005). Communicative skills in relation to gender, birth order, childcare and socioeconomic status in 18month-old children. Scandinavian journal of psychology, 46(6), 485-491. https://doi.org/10.1111/j.1467-9450.2005.00480.x 
Bolte, L., y Rojas, P. (2013). Trastornos del habla y del lenguaje infantil: ¿cómo lo abordamos? Parte II. Centro Psicológico CPC. https://www.centropsicologicocpc.es/trastornos-del-habla-y-del-lenguajeinfantil-como-los-abordamos

Bonilla Hermenegildo, J. P., y Reyes Figueroa, C. D. L. Á. (2017). Influencia de la disfasia en la calidad del desarrollo del nivel expresivo del lenguaje oral en los niños de 5 a 6 años [Bachelor's thesis, Universidad de Guayaquil Facultad de Filosofía, Letras y Ciencias de la Educación].

Campos, A. L. (2010). Primera infancia: una mirada desde la neuroeducación. Lima: Cerebrum y OEA.

Choudhury, N., y Benasich, A. (2003). A family aggregation study: The influence of family history and other risk factors on language development. Journal of Speech, Language, and Hearing Research, 46(2), 261-272. https://doi.org/10.1044/1092-4388(2003/021)

Morán Gómez, Y. A. (2020). Neurodidáctica para la construcción de aprendizajes en educación preescolar [Doctoral dissertation, Benemérita y Centenaria Escuela Normal del Estado de San Luis Potosí].

NIH. (2019). El trastorno específico del lenguaje. Recuperado el 07 de 10 de 2020, de Hoja de información del NIDCD. Voz, habla y lenguaje. https://www.nidcd.nih.gov/sites/default/files/Content\%20Images/SpecificLangu agelm prmntSP-508.pdf

Peñaloza, C. (2018). Habilidades lingüístico-discursivas y características familiares y socioambientales de niños preescolares de Ciudad de México [tesis doctoral]. Universidad Nacional Autónoma de México.

Portellano, J.A. (2018). Neuroeducación y funciones ejecutivas. Madrid: Cepe.

Román, F., y Poenitz, V. (2018). La neurociencia aplicada a la educación: aportes, desafíos y oportunidades en América Latina. RELAdEI. Revista Latinoamericana de Educación Infantil, 7(1), 88-93.

Salvatierra-Vera, N. M., y Zambrano-Montes, L. C. (2021). Estrategias de aprendizaje en niños y niñas con trastorno específico del lenguaje. Polo del Conocimiento, 6(1), 760-779.

Secretaria de Educación Pública de México. (2018). Plan de intervención en el aula para alumnos con dificultades en la comunicación. https://www.ceviedgespe.com/documentos/0855.pd 
Uzcátegui, A., Martínez, C., Méndez, L., y Pantoja, J. (2007). Estudio epidemiológico de los trastornos del aprendizaje en escolares, en una consulta de neuropediatría. Archivos Venezolanos de Puericultura y Pediatría, 70(3), 81$88 . \quad$ http://ve.scielo.org/scielo.php?script=sci arttext\&pid=S000406492007000300003\&lng=es\&tlng=es

Villanueva, P., De Barbieri, Z., y Palomino, H. (2008). Alta prevalencia de trastorno específico de lenguaje en isla Robinson Crusoe y probable efecto fundador. Revista médica de Chile, 2, 186-192. 